\title{
Welcoming Remarks by the Chancellor of the University of California
}

Welcome to IAU Colloquium 152 on EUV Astrophysics.

Many of you have come a long way to participate in this conference; from Russia, Japan, England, Germany, Italy and many other countries. You are the experts but I would like to remind you of some of the milestones in this field, and in particular, Berkeley's role in the development of EUV astrophysics. Twenty years ago we had no EUV sources. From 1959 when the first papers were published on this subject until 1974, everyone assumed that interstellar space was filled with gas that would be entirely opaque to EUV radiation. In 1974, a Berkeley group, led by Stu Bowyer, published an article in the Astrophysical Journal. The article predicted that conventional wisdom was wrong and that space was not nearly as opaque in the EUV as predicted.

The only way to know was to look. Within a year, Bowyer and his group submitted a winning proposal to NASA for an EUV mission. Unfortunately, the mission did not get very far before it was terminated; fortunately, Stu was not easily discouraged. In less than a year he found a way to fly a small EUV telescope on the joint US-Soviet ApolloSoyuz mission. On that mission, the Berkeley group discovered four EUV emitting stars. Based on that success, the group sent another proposal to NASA. That proposal was selected for development in 1976. But it did not fly until June of 1992 when the Extreme Ultraviolet Explorer mission finally got off the ground.

Was it worth the wait? I think so. Here you are today, about to start the first major international conference dedicated to exploring this new spectral window. The window was opened by that long-awaited launch.

Let me congratulate Stu Bowyer for never giving up and thereby bringing about today's proceedings. His work is indicative of the quality of research that goes on here at Cal.

It is with great pride that I welcome you to the University of California at Berkeley. Thank you and I wish you well in your discussions!

Chang-Lin Tien, Chancellor University of California, Berkeley 